\title{
THE USE OF SIMULATION TO MODEL THE DISPATCH OF INBOUND CONTAINERS IN PORT TERMINALS
}

\author{
Martin Guillermo Cornejo Sarmiento ${ }^{1}$, Eugenio Kahn Epprecht ${ }^{1}$,

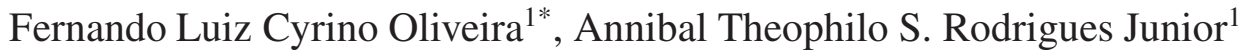 \\ and Smith Washington Arauco Canchumuni²
}

Received April 05, 2018 / Accepted January 30, 2019

\begin{abstract}
This paper describes a study of the dispatch planning/scheduling process for inbound containers handled with a reach stacker. Client container pickup is scheduled at least one day in advance for one of six two-hour time windows (six five-container-high stacks per time window) on a given day. A buffer area is available for the containers to be moved in when clients are being served. The aim of this study was to determine the conditions required to ensure that all the containers are dispatched within the scheduled time window and so meet the clients' requirements. To this end, the performance indicators were identified and compared using simulations as an analytical tool. The results indicate that the shortest-processing-time (SPT) queueing discipline is preferable to the first-come-first-served (FCFS) discipline and that client arrivals can usefully be restricted to periods shorter than two hours in order to meet container-dispatch and service-quality objectives.
\end{abstract}

Keywords: simulation, queueing disciplines, inbound container management, container stacking, time windows.

\section{INTRODUCTION}

The introduction and development of the container as the basis of a unit load concept for cargo and as a means of increasing efficiency during cargo handling posed a serious challenge for those responsible for planning port activities. This new concept brought about a major revolution in cargo transport, as more convenient, safer and standardized cargo operations led to cost reductions and facilitated many aspects of these operations. Standardized handling equipment was also

*Corresponding author - https://orcid.org/0000-0003-1870-9440

${ }^{1}$ Departamento de Engenharia Industrial, Pontifícia Universidade Católica do Rio de Janeiro, Rua Marquês de São Vicente, 225, Gávea, 22451-900 Rio de Janeiro, RJ, Brasil.

E-mails: martin.cornejo8@gmail.com; eke@puc-rio.br; cyrino@puc-rio.br; annibalrodriguesjr@yahoo.com.br

2 Departamento de Engenharia Elétrica, Pontifícia Universidade Católica do Rio de Janeiro, Rua Marquês de São Vicente, 225, Gávea, 22451-900 Rio de Janeiro, RJ, Brasil. E-mail: saraucoc@uni.pe 
adopted for moving and storing containers, thereby reducing costs through the standardization of processes. The introduction of containers thus increased the productivity of both terminals and ships (Lee et al., 2008; Bernhofen et al., 2016; Levinson, 2016).

Container terminals are an increasingly important link in the supply chain of a wide range of productive sectors. Statistics such as those on container port traffic by country between 2000 and 2016 (The World Bank, 2018) and in Brazil between 2007 and 2017 (Abratec, 2018) show that there has been significant growth in container traffic in Brazil and the rest of the world in recent years, justifying the many academic studies on logistics in port terminals. To meet the challenges this growth poses, any idle capacity in terminal facilities must be constantly identified and operational efficiency sought by means of daily decision-making and efficient medium- and long-term planning (Moorthy \& Teo, 2006; Cordeau et al., 2007; Vacca et al., 2007; Novaes et al., 2012; Bearzotti et al., 2013; Wilmsmeier et al., 2013; Pereira et al., 2018).

The need to take daily operational decisions, together with the unique, complex dynamics involved, makes terminal container management quite unlike management of any other aspects of cargo handling/storage. Problems commonly found in terminals have been exhaustively studied, and the lines of research developed to address these have been discussed in the literature. Some authors have also made significant contributions in the form of surveys of container terminal operations (Steenken et al., 2004; Murty et al., 2005; Günther \& Kim, 2006; Crainic \& Kim, 2007; Stahlbock \& Voß, 2008; Carlo et al., 2014a). The use of simulation is a recurring feature of all these studies [e.g., Hartmann (2004)] and is a result of the level of uncertainty in terminal operations. One of the processes that contribute to this uncertainty is the arrival of trucks to pick up inbound (import) containers. The issues addressed in these studies include decisions related to operational strategies, such as identification of the best yard layout, container stacking strategy, stack geometry, dispatching policy and appointment times (de Castilho \& Daganzo, 1993; Kim \& Kim, 1999; Vis \& De Koster, 2003; Dekker et al., 2006; Junqueira et al., 2010; Borgman et al., 2010; Roy \& De Koster, 2012; Carlo et al., 2014b). As an aid to the management and control of these processes, various key indicators are used to quantify the relative performance of each process. Murty et al. (2005) describe in considerable detail indicators for each of the processes involved in the operation of a container terminal.

Reach stackers are widely used in small terminals. Our study seeks to compensate to a certain extent for the relative inefficiency of these stackers compared with their more expensive vertical counterparts by modeling and adopting more efficient processes that can increase their productivity. Container dispatch using queueing policies and reach stackers was studied by Rodrigues Junior (2009) in the specific context of a Brazilian container terminal. Our literature review also identified a study of inbound container pickup by Sgouridis \& Angelides (2002), although their study differs from the present article in that neither reach stackers nor container pre-segregation was used; instead, the containers were retrieved or stacked in different parts of the yard with vertical-access equipment. Kozan \& Casey (2007) discuss the (more complex) optimization of a simulation model of a multimodal (inbound and outbound) container terminal using verticalaccess and side-access equipment. 
We consider the container dispatch process described by Rodrigues Junior (2009). The aim of the present study is to determine the feasibility of dispatching all the containers scheduled within each two-hour time window. To this end, we first compare two sets of queueing disciplines to determine which one produces the best performance in terms of both making the dispatch operation less likely to exceed the time window and offering the shortest mean truck waiting time and service time. We then suggest what conditions can be useful to ensure that this target of completing dispatch operations within the time windows is achieved. The findings of this study can therefore be expected to find widespread application in the many facilities where reach stackers are used.

The remainder of this article is organized as follows: Section 2 discusses the container dispatch process. Section 3 introduces the variables and parameters used in the simulation model, and Section 4 discusses the simulation model itself. The results of the simulations are described and discussed in Section 5, and the general conclusions are given in Section 6.

\section{THE CONTAINER DISPATCH PROCESS}

\subsection{Definition of the environment and operational assumptions}

Only reach stackers are used in the process studied here. As noted earlier, this type of equipment is used mainly in smaller terminals, where its popularity is due to its low cost and the fact that it has greater mobility than vertical access equipment. However, reach stackers suffer from a disadvantage in that they require a greater number of unproductive (or reshuffling) moves to reach a given container because they access stacks from the side.

If time windows were not used, customers could arrange in advance to collect their containers as they are cleared by the customs authorities. The terminal would not require that customers' containers be collected within a particular period (time window); instead, containers could be collected throughout the day. However, in the final analysis this would lead to poor use of resources because of fluctuating usage levels and operational unpredictability.

Vis et al. (2005) and Murty et al. (2005) discuss the use of time windows for delivery of outbound (export) containers and pickup of inbound ones. They note that appointments in time windows make operational planning in terminals easier and that simple online rules for making these appointments must be developed to minimize truck waiting time and congestion on the roads in the terminal. In the present paper we therefore sought to assess the impact of using time windows in the process studied.

Below we describe the operational policies for the process and give details of the characteristics of the reach stackers. These definitions come from Rodrigues Junior (2009), and correspond to the processes of a real container terminal.

a) import clients arrange at least one day in advance for their containers to be picked up in one of various predetermined time windows (six two-hour time intervals, as shown in Figure 1); 
b) containers that are to be picked up the next day in the various time windows must be moved to a specific area the night before the scheduled pickup;

c) a maximum of thirty containers in six five-container-high stacks can be scheduled in each time window;

d) trucks must arrive at the facility before the end of the previously scheduled two-hour time window; and it must be pursued that the trucks will be served within this time window;

e) access to the stacks shall be from both sides so that the reach stacker's performance can be optimized (to understand what is meant by "side", see the indications "Far right" and "Far left" of the initial buffer area in Figure 1);

f) a buffer area will be designated at one side of the stacking area. This area shall be the same size as the area corresponding to one time window (i.e., it will be able to hold up to thirty containers) so that containers that are not being picked up can be moved around. To improve efficiency, this buffer area shall be moveable and shall follow the active window, i.e., it will occupy the area of the block just emptied in the previous time window, as shown in Figure 1; and

g) only one reach stacker will be used. The reach stacker can move only one container and can stack it on (or remove it from) the allocated stack on the far right or left of the container block (side access).

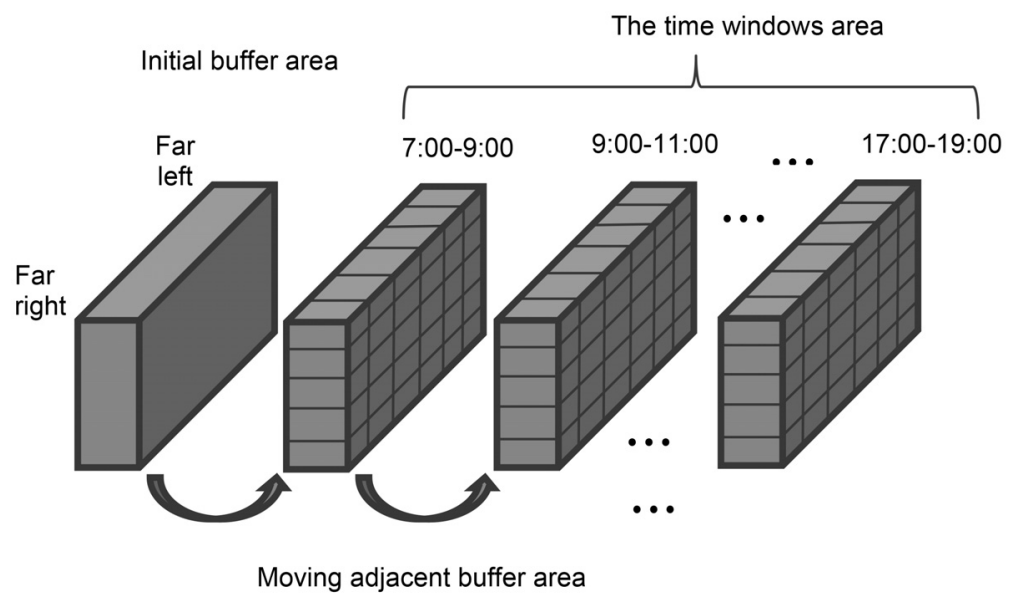

Figure 1 - The container storage yard and the arrangement of blocks (six two-hour time windows area and a moving adjacent buffer area).

The model used here assumes that for a specific container to be removed from a block (the set of stacks of containers in a particular area, which can be a time-window area or the adjacent buffer area) all the containers in front of it or above it (containers not scheduled to be picked up) must 
have been removed. Containers not scheduled to be picked up (unproductive moves) should be moved from the block where the target container is (e.g. the time-window area) to the same side (right or left) of the transfer block (e.g. the buffer area) and stacked there unless that side is already full. Note that the next target container may be in the buffer area as a result of previous movements and in this case the containers that should be moved to clean the access to it would be moved to the time window area. In any case, they should be moved to the same side (right or left) of the time window area, unless that side is already full. This ensures that the reach stacker moves as little as possible. See Figure 1.

\subsection{Queueing disciplines}

The time required to access and dispatch a particular container (service time) is proportional to the number of moves needed, i.e., the number of containers that have to be moved to reach it. In light of this, we analyzed the servicing process using two queueing disciplines: shortest processing time (SPT) and first come, first served (FCFS). In the process considered here, under the SPT queueing discipline the truck whose container requires the fewest moves (of all the containers allocated for pickup by trucks that have arrived) is served first.

For clarity, we illustrate the operational policies indicated above with an example of stacking/unstacking operations under the FCFS queueing discipline. Figure 2 shows the initial stacking arrangement in a given time window and the final arrangement together with the corresponding buffer area after two containers have been retrieved. The container corresponding to the first truck to arrive, which is identified as number 22, is in the time-window area (Original Window) in the position (height 4, stack 3). Seventeen moves are needed to retrieve it from the right, while only twelve are needed to retrieve it from the left. The container is therefore retrieved from the left because this requires the fewest moves. The eleven containers that are not required are ustacked from the Original Window in order to clear the access to container \#22 and stacked in Buffer Area 1 in the reverse order to that in which they were originally stacked. The situation is then as shown in Modified Window 1 and Buffer Area 1. The second arrival is of truck \#27. The corresponding container is in Buffer Area 1 (height 1, stack 3) and is retrieved from the right. Four unproductive moves (containers 10, 24, 11 and 3) and one productive move (container 27) are required. Note that the right-hand side of Modified Window 1 (to which the unwanted containers need to be transferred) is full. Because of this exceptional situation, the containers are moved to the left of the window. Modified Window 2 and Buffer Area 2 show the final arrangement of the containers after containers nos. 22 and 27 have been retrieved. The retrieval process continues in this way until all thirty containers have been dispatched.

\section{SIMULATION MODEL}

\subsection{Arrival process}

The most common assumption for the arrival rate is that clients arrive according to a Poisson distribution or, equivalently, that the variable inter-arrival time has an exponential distribution. 


\begin{tabular}{|c|c|c|c|c|c|c|c|}
\hline & \multicolumn{6}{|c|}{ STACK № } \\
\hline & & 1 & 2 & 3 & 4 & 5 & 6 \\
\hline \multirow{5}{*}{$\begin{array}{l}\text { 동 } \\
\text { 포 }\end{array}$} & 5 & 27 & 14 & 6 & 10 & 13 & 15 \\
\hline & 4 & 3 & 5 & 22 & 19 & 26 & 20 \\
\hline & 3 & 11 & 7 & 1 & 21 & 23 & 2 \\
\hline & 2 & 24 & 28 & 4 & 29 & 9 & 30 \\
\hline & 1 & 10 & 8 & 25 & 12 & 17 & 16 \\
\hline
\end{tabular}

\begin{tabular}{|c|c|c|c|c|c|}
\hline \multicolumn{6}{|c|}{ The container dispatch process (FCFS discipline) } \\
\hline $\begin{array}{c}\text { Dispatch } \\
\text { no. }\end{array}$ & $\begin{array}{c}\text { Truck } \\
\text { no. }\end{array}$ & $\begin{array}{c}\text { From the } \\
\text { right }\end{array}$ & $\begin{array}{c}\text { From the } \\
\text { left }\end{array}$ & $\begin{array}{c}\text { Side } \\
\text { access }\end{array}$ & $\begin{array}{c}\text { no. of } \\
\text { moves }\end{array}$ \\
\hline 1 & 22 & 17 & 12 & LEFT & 12 \\
\hline 2 & 27 & 5 & 11 & RIGHT & 5 \\
\hline
\end{tabular}

\begin{tabular}{|c|c|c|c|c|c|c|c|c|}
\hline \multirow{2}{*}{\multicolumn{2}{|c|}{\begin{tabular}{l|}
$\tilde{N}$ \\
$\tilde{\sim}$ \\
$\underline{\underline{J}}$
\end{tabular}}} & & \multicolumn{6}{|c|}{ STACK NNo } \\
\hline & & & 1 & 2 & 3 & 4 & 5 & 6 \\
\hline \multirow{6}{*}{$\leq$} & \multirow{5}{*}{$\begin{array}{l}\text { 도 } \\
\text { 顽 }\end{array}$} & 5 & - & - & - & 10 & 13 & 15 \\
\hline & & 4 & - & - & - & 19 & 26 & 20 \\
\hline & & 3 & - & - & 1 & 21 & 23 & 2 \\
\hline & & 2 & - & - & 4 & 29 & 9 & 30 \\
\hline & & 1 & - & - & 25 & 12 & 17 & 16 \\
\hline & & & & & 17 & TV & & \\
\hline
\end{tabular}

\begin{tabular}{|c|c|c|c|c|c|c|c|}
\hline & \multicolumn{6}{|c|}{ STACK No } \\
\hline & & 1 & 2 & 3 & 4 & 5 & 6 \\
\hline \multirow{5}{*}{$\begin{array}{l}\text { 돔 } \\
\text { 폰 }\end{array}$} & 5 & & 8 & 10 & & & \\
\hline & 4 & & 28 & 24 & & & \\
\hline & 3 & 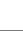 & 7 & 11 & & & \\
\hline & 2 & & 5 & 3 & & & \\
\hline & 1 & 6 & 14 & 27 & & & \\
\hline
\end{tabular}

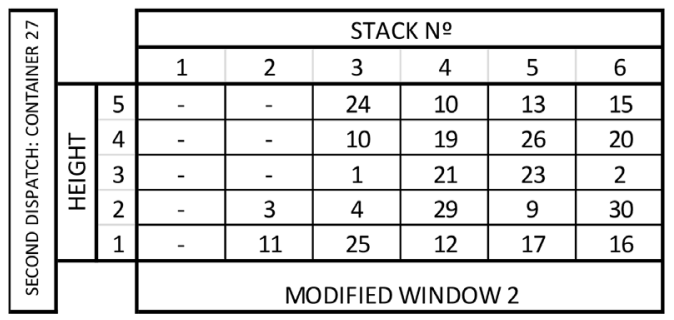

\begin{tabular}{|c|c|c|c|c|c|c|c|}
\hline & \multicolumn{6}{|c|}{ STACK № } \\
\hline & & 1 & 2 & 3 & 4 & 5 & 6 \\
\hline \multirow{5}{*}{$\begin{array}{l}\text { 战 } \\
\frac{\mathrm{J}}{\mathrm{I}}\end{array}$} & 5 & & 8 & - & & & \\
\hline & 4 & & 28 & - & & & \\
\hline & 3 & & 7 & - & & & \\
\hline & 2 & & 5 & - & & & \\
\hline & 1 & 6 & 14 & - & & & \\
\hline
\end{tabular}

Figure 2 - The logic involved in moving the containers in the stacks under the FCFS queueing discipline (this example shows the first two dispatches).

However, for the process studied here, this assumption is not valid as a fixed number of trucks (thirty) is assigned to each time window and, hence, the arrival events in each time window are not independent.

We therefore adopt two alternative arrival models, which, although involving some assumptions that can only be considered as approximations, represent extreme scenarios in terms of the distribution of arrivals over time. In the first scenario the arrival times follow a uniform distribution within the time window. If the start of the time window is represented by $t=0$ and the end by $t=120$ (in minutes), the arrival time for each truck is a random variable $T(T \sim U[0,120])$.

In the other scenario, arrivals are assumed to be concentrated at the beginning of the window and, as the truck drivers are all independent and unaware of each other's movements, the inter-arrival times are assumed to be distributed approximately exponentially. However, the inter-arrival time should be such that the probability of the 30th truck arriving at time $t>120$ (or, equivalently, the probability of the thirty trucks not arriving before the end of the 120-minute time window) is very small. To this end, the inter-arrival times are generated according to an exponential distribution but after the 30th arrival no more trucks arrive and the process stops. If until time $t=120$ fewer than 30 trucks have arrived, the run is discarded. However, as the probability of this happening 
should be very small, the number of discarded runs should be very small too. Table 1 is used to choose the expected inter-arrival time $(1 / \lambda$, where $\lambda$ is the corresponding parameter). It shows the relationship between four possible values $(1 / \lambda=\{4.0,3.5,3.0,2.5\}$ minutes/arrival $)$ and the probability of the 30 trucks not arriving before the end of the 120 minutes. A value of $1 / \lambda=2.5$ minutes/arrival (option 4) was chosen as it produced a probability of less than $1 \%$.

Table 1 - Relationship between four expected values of inter-arrival time $1 / \lambda$ (in minutes/arrival and assuming an exponential distribution) and the probability of the thirty trucks not arriving before the end of the 120-minute time window $(P(X<30))$.

\begin{tabular}{l|c|c|c|c|c|c}
\hline \multirow{2}{*}{ Variable } & Assumed & \multirow{2}{*}{ Mean } & \multicolumn{5}{|c}{ Options } \\
\cline { 5 - 8 } & distribution & & 1 & 2 & 3 & 4 \\
\hline Inter-arrival time (in min) & Exponential & $1 / \lambda$ & 4.0 & 3.5 & 3.0 & 2.5 \\
\hline no. of arrivals in 1 min & Poisson & $\lambda$ & 0.25 & 0.29 & 0.33 & 0.40 \\
\hline$X:$ Number of arrivals in a & Poisson & $120 \lambda$ & 30 & 34.29 & 40 & 48 \\
$\begin{array}{l}120-\text { minutes window } \\
\text { Probability that } X \text { is less than } 30(P(X<30))\end{array}$ & $47.57 \%$ & $20.95 \%$ & $4.32 \%$ & $0.22 \%$ \\
\hline
\end{tabular}

\subsection{Container move time}

Service time in the case studied here depends essentially on the time it takes to move containers using a single agent (a reach stacker) so they can be dispatched to clients. Container move time corresponds to the time taken to remove a container from a stack and place it on a truck (a productive move from the time-window area or buffer area to a truck) or the time taken to remove a container from a stack and place it on another stack (an unproductive move from the time-window area to the buffer area or vice versa).

In this study we assume that the container move time (the time for a productive or unproductive move) has a normal distribution with a mean of 2.5 minutes and standard deviation of 0.1667 minutes. These values were obtained by Rodrigues Junior (2009) in an interview with the operations department at a port in Brazil.

\subsection{Simulation dynamics}

Our analysis focuses on the dispatch of each two-hour window. Truck arrivals and container moves were simulated in Matlab. The maximum number of containers to be picked up in each time window was assumed to be 30 . The algorithm has the following main functions:

a) shuffling of the order of trucks arrivals to ensure random arrivals;

b) shuffling of the containers so they are randomly distributed in the six five-container-high stacks;

c) simulation of the reach stacker operations and all the logic governing the movement of the containers in the stacks (blocks). In the case of the FCFS queueing discipline, the moves 
needed to remove the containers in the same order as the corresponding trucks arrive must be analyzed. In the case of the SPT queueing discipline, the container that requires the fewest moves is selected from the containers corresponding to the trucks in the queue. This requires:

c1) generation of random times of truck arrivals, while ensuring that all 30 trucks arrive within the time interval defined for each window. Two alternative assumptions regarding this variable were made (see Section 3.1): client arrival times have a uniform distribution $(U[0,120])$ or the inter-arrival time has an exponential distribution with an expected value of 2.5 minutes/client;

c2) generation of random move times for each container. These include unproductive moves (to get access to the container that is to be picked up) as well as productive moves (to retrieve the container that is to be picked up). According to historical records (see Section 3.2), this variable has a normal distribution $[N(2.5 ; 0.1667)]$.

\subsection{Performance indicators}

The following performance indicators, which are based on the dispatch of two-hour windows, were analyzed:

a) Time in excess of $\mathbf{1 2 0}$ minutes $(\mathrm{Te})$. This key variable indicates whether the stipulated two-hour window is sufficient to ensure that thirty trucks can be served. It is equal to the difference between the moment the last client was served and the end of the 120-minute window;

b) Cumulative arrival time. The time between the start of the window and the moment the last client (truck) arrives;

c) Number of clients by waiting time for five different 30-minute bands. This shows how the 30 clients in each window are distributed in terms of waiting time in the queue $(W)$ for five 30-minute bands ( $W \leq 30 ; 30<W \leq 60$; $60<W \leq 90 ; 90<W \leq 120$; and $W>120$ ). This information complements Mean waiting time (in the queue) per truck and Mean queue size;

d) Mean number of moves to access a container and Mean service time per truck (excluding waiting time);

e) Number of clients for which dispatching finished after the end of the time window, i.e., the number of clients served after the 120-minute window; and, lastly,

f) Total equipment idle time, which includes reach-stacker idle time at the beginning of each window, between clients served and when the process is completed before the end of the two-hour window. 


\section{RESULTS AND DISCUSSION}

Five hundred replications were used (a replication corresponds to the process of serving 30 clients in a window). Therefore, according to the central limit theorem, the estimated mean value of any of the performance indicators considered should have an error of less than $10 \%$ of its standard deviation at a $95 \%$ confidence level.

The following sections define the different values of parameters considered, and the results for the indicators for the two simulations performed are then analyzed. In the first simulation we sought to determine what queueing discipline would best satisfy the objective of efficiently dispatching all the containers scheduled for each window, while in the second, client arrivals were limited to time windows of less than two hours in an attempt to achieve the same objective.

To enable the scenarios evaluated in the simulations to be compared more effectively, the same truck (client) arrival order and same initial container layout were used for the 500 time-window replications. In other words, the same two sets of random numbers generated in the simulation algorithm specified in (a) and (b) in Section 3.3 were used in the different scenarios. However, the two sets of values for the variables in the simulation model referred to in (c1) and (c2) were different.

\subsection{Comparative analysis of the queueing disciplines}

The SPT and FCFS queueing disciplines were analyzed in both the scenarios shown in Table 2 . These scenarios (A and B) differ in terms of the probability distribution that the arrival times are assumed to have. The parameters and the distributions of the simulation variables shown in Table 2 were defined in Section 3.

Table 2 - Values of parameters in the first simulation: comparison of the queueing disciplines.

\begin{tabular}{|c|c|c|c|c|c|}
\hline \multirow{4}{*}{ Simulation variables } & \multirow{4}{*}{ Units } & \multicolumn{4}{|c|}{ Queueing disciplines } \\
\hline & & SPT & FCFS & SPT & FCFS \\
\hline & & \multicolumn{4}{|c|}{ Scenarios evaluated } \\
\hline & & $\mathrm{A}$ & $\mathrm{A}$ & $\mathrm{B}$ & $\mathrm{B}$ \\
\hline Arrival models & & \multicolumn{2}{|c|}{ Inter-arrival time } & \multicolumn{2}{|c|}{ Arrival time } \\
\hline Distribution & \multirow[b]{2}{*}{$\begin{array}{l}\text { minutes/arrival or } \\
\text { minutes }\end{array}$} & \multicolumn{2}{|c|}{$\begin{array}{c}\text { Exponential }(\lambda) \\
\operatorname{Exp}(\lambda=0.4)\end{array}$} & \multicolumn{2}{|c|}{$\begin{array}{c}\text { Uniform } \\
U[0,120]\end{array}$} \\
\hline Mean & & $\begin{array}{c}2.5 \\
-\end{array}$ & $\begin{array}{c}2.5 \\
-\end{array}$ & $\begin{array}{l}- \\
60\end{array}$ & $\begin{array}{l}- \\
60\end{array}$ \\
\hline \multicolumn{6}{|l|}{ Container move time } \\
\hline \multirow{3}{*}{$\begin{array}{l}\text { Distribution } \\
\text { Mean }(\mu) \\
\text { Standard deviation }(\sigma)\end{array}$} & \multirow{3}{*}{ minutes/container } & \multicolumn{4}{|c|}{ Normal $(N(\mu, \sigma))$} \\
\hline & & 2.5 & 2.5 & 2.5 & 2.5 \\
\hline & & 0.1667 & 0.1667 & 0.1667 & 0.1667 \\
\hline Dispatch time window & minutes & 120 & 120 & 120 & 120 \\
\hline Arrival time window & minutes & 120 & 120 & 120 & 120 \\
\hline
\end{tabular}




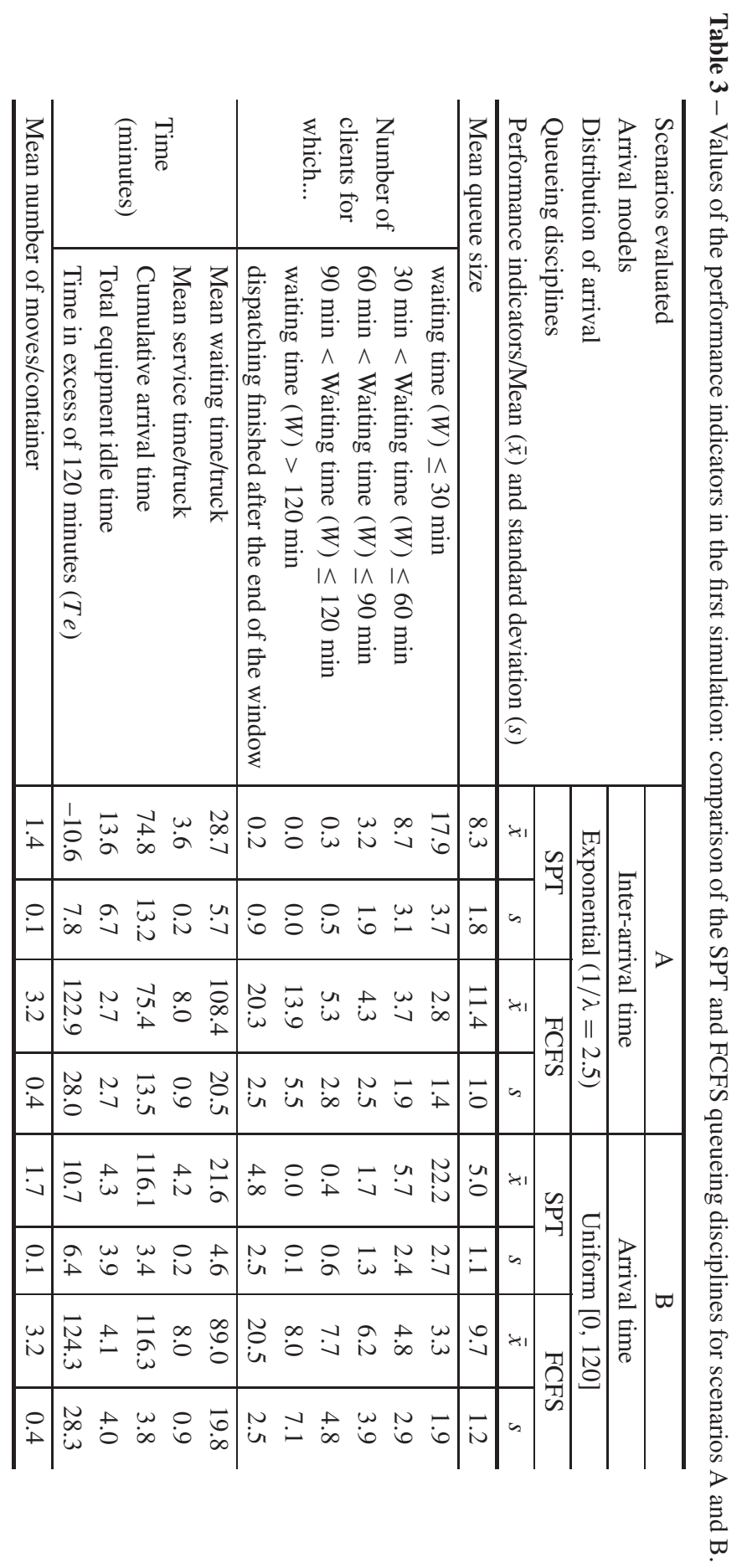

Pesquisa Operacional, Vol. 39(1), 2019 
Table 3 shows the results for the indicators in the simulation to evaluate the two queueing disciplines. Note that, overall, the SPT queueing discipline performed significantly better than the FCFS queueing discipline in both scenarios. For example, in scenario B and considering average values, 28 of the clients (93\%) would be served within one hour or less with the SPT discipline, while the corresponding figure for the FCFS discipline is 8 (26.9\%). Figure 3 shows the mean percentage of trucks served and the corresponding waiting times $(W)$ in 30-minute intervals for both scenarios. Complementing this, for scenario B and considering average values, the mean waiting time/truck under the SPT queueing discipline and FCFS queueing discipline is 21.6 and 89.0 minutes, respectively, and the mean service time/truck is 4.2 and 8.0 minutes, respectively. These results show that the SPT queueing discipline results in a better service level and improved customer satisfaction because of the reduced waiting time and service time.

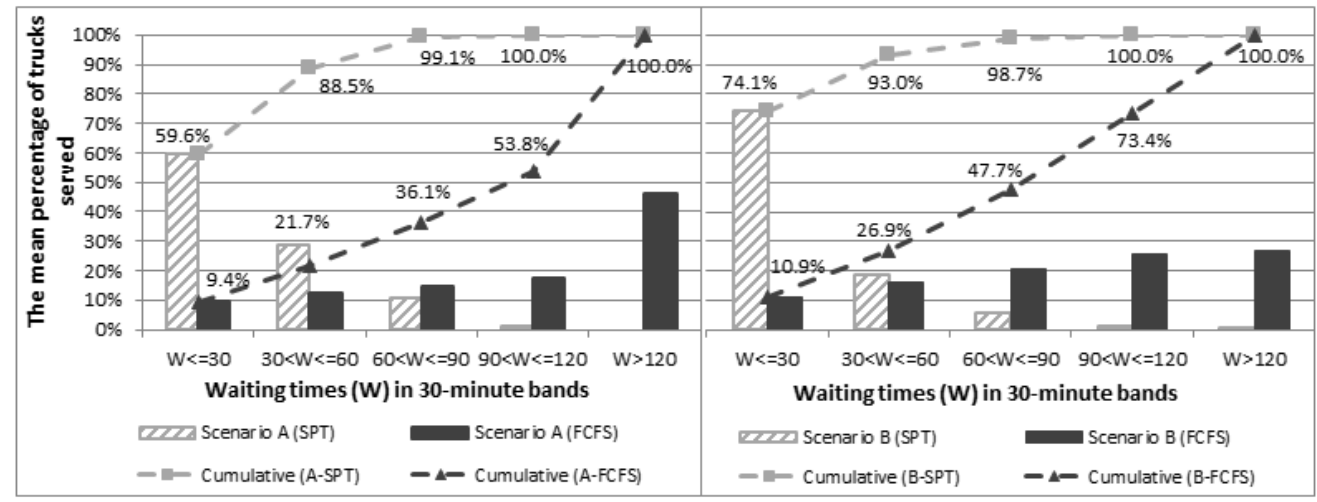

Figure 3 - Comparison of the mean percentage of trucks served and their waiting times $(W)$ in 30-minute bands under the SPT and FCFS queueing disciplines (scenarios A and B).

Once again confirming the superiority of the SPT queueing discipline, Figure 4 shows a comparison of the percentage of replications and their corresponding duration in terms of the Time in Excess of 120 Minutes ( $\mathrm{Te}$ ) in 30-minute bands under the SPT and FCFS disciplines for scenarios A and B. Taking an example, in scenario A, the percentage of replications in which 30 trucks are served before the end of the two-hour time window $(T e \leq 0)$ is $92.2 \%$ under the SPT queueing discipline and only $0.2 \%$ under the FCFS discipline. The percentage of replications with a maximum duration of 180 minutes $(T e \leq 60)$ is $100.0 \%$ under the SPT discipline and only $1.0 \%$ with the FCFS discipline.

The superior performance of the SPT queueing discipline in both scenarios can also be observed in the results for the other variables in Table 3. On the basis of this significantly superior performance it was decided to focus exclusively on the SPT queueing discipline and to investigate the possibility of achieving the performance objectives with this discipline. The values of the variable Time in Excess of 120 Minutes ( $T e$ ) are analyzed in detail because they are of particular importance in any assessment of the feasibility of dispatching 30 containers within each scheduled time window. In scenario A, the mean of $T e$ is -10.6 minutes (i.e., the process finishes on 


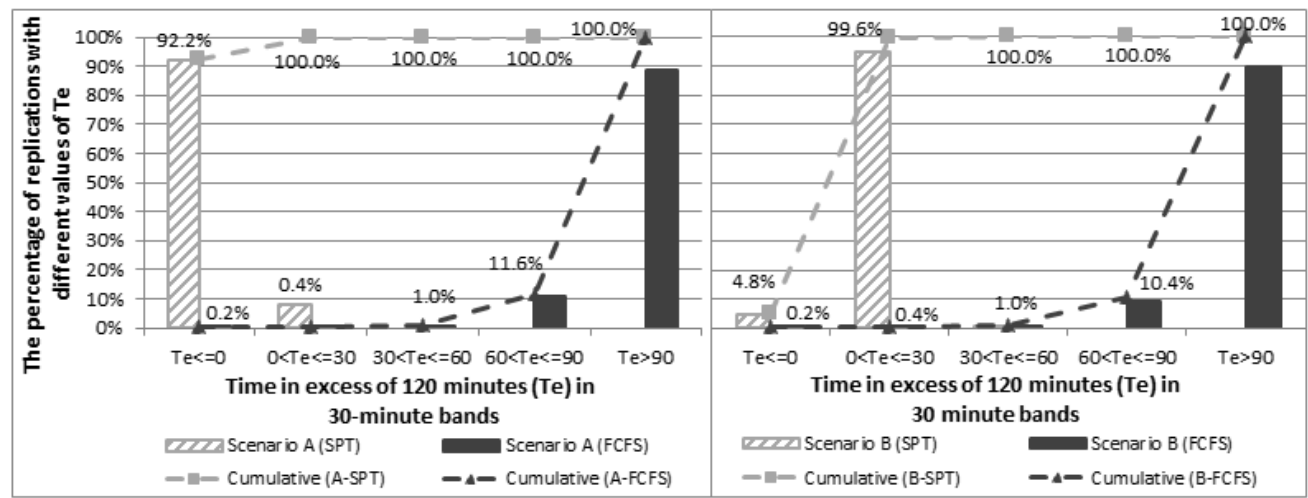

Figure 4 - Comparison of the percentage of replications and their corresponding duration (Time in excess of 120 minutes, $T e$ ) under the SPT and FCFS queueing disciplines (scenarios A and B).

average 10.6 minutes before the end of the two-hour window) with a standard deviation of 7.8 minutes, and in scenario B it is 10.7 minutes (i.e., the process finishes on average 10.7 minutes after the end of the two-hour window) with a standard deviation of 6.4 minutes.

In addition, Table 4 shows the percentage of replications by $T e$ in five-minute bands for scenarios $\mathrm{A}$ and $\mathrm{B}$. The values of $T e$ are shown in graph form in Figure 5. The decision to impose additional conditions or restrictions is based on the probabilities of exceeding the two-hour limit. These can be estimated using the cumulative percentages in Table 4. For example, the estimated probability of serving 30 trucks before the end of the window $(T e \leq 0)$ in scenarios A and B is 0.922 and 0.048 , respectively, and the estimated probability of serving the trucks in 130 minutes or less $(T e \leq 10)$ is 0.986 and 0.460 , respectively.

Table 4 - Percentage of replications and their corresponding duration (expressed as $T e$, Time in Excess of 120 Minutes, in five-minute bands) under the SPT queueing discipline for scenarios A and B.

\begin{tabular}{|c|c|c|c|c|c|c|}
\hline Scenarios & \multicolumn{3}{|c|}{$\bar{A}$} & \multicolumn{3}{|c|}{$\mathrm{B}$} \\
\hline Arrival models & \multicolumn{3}{|c|}{ Exponential $(1 / \lambda=2.5)$} & \multicolumn{3}{|c|}{ Uniform $(U[0,120])$} \\
\hline $\begin{array}{l}\text { Five-minute } \\
\text { bands of } T e\end{array}$ & $\begin{array}{c}\text { Number } \\
\text { of } \\
\text { replications }\end{array}$ & $\begin{array}{l}\text { Percentage } \\
\text { of } \\
\text { replications }\end{array}$ & $\begin{array}{c}\text { Cumulative } \\
\text { percentage of } \\
\text { replications }\end{array}$ & $\begin{array}{c}\text { Number } \\
\text { of } \\
\text { replications }\end{array}$ & $\begin{array}{l}\text { Percentage } \\
\text { of } \\
\text { replications }\end{array}$ & $\begin{array}{l}\text { Cumulative } \\
\text { percentage of } \\
\text { replications }\end{array}$ \\
\hline$T e \leq 0$ & 461 & $92.2 \%$ & $92.2 \%$ & 24 & $4.8 \%$ & $4.8 \%$ \\
\hline $0<T e \leq 5$ & 24 & $4.8 \%$ & $97.0 \%$ & 63 & $12.6 \%$ & $17.4 \%$ \\
\hline $5<T e \leq 10$ & 8 & $1.6 \%$ & $98.6 \%$ & 143 & $28.6 \%$ & $46.0 \%$ \\
\hline $10<T e \leq 15$ & 5 & $1.0 \%$ & $99.6 \%$ & 157 & $31.4 \%$ & $77.4 \%$ \\
\hline $15<T e \leq 20$ & 2 & $0.4 \%$ & $100 \%$ & 68 & $13.6 \%$ & $91.0 \%$ \\
\hline$T e>20$ & 0 & $0.0 \%$ & $100 \%$ & 45 & $9.0 \%$ & $100 \%$ \\
\hline Total (replications) & 500 & $100 \%$ & - & 500 & $100 \%$ & - \\
\hline
\end{tabular}



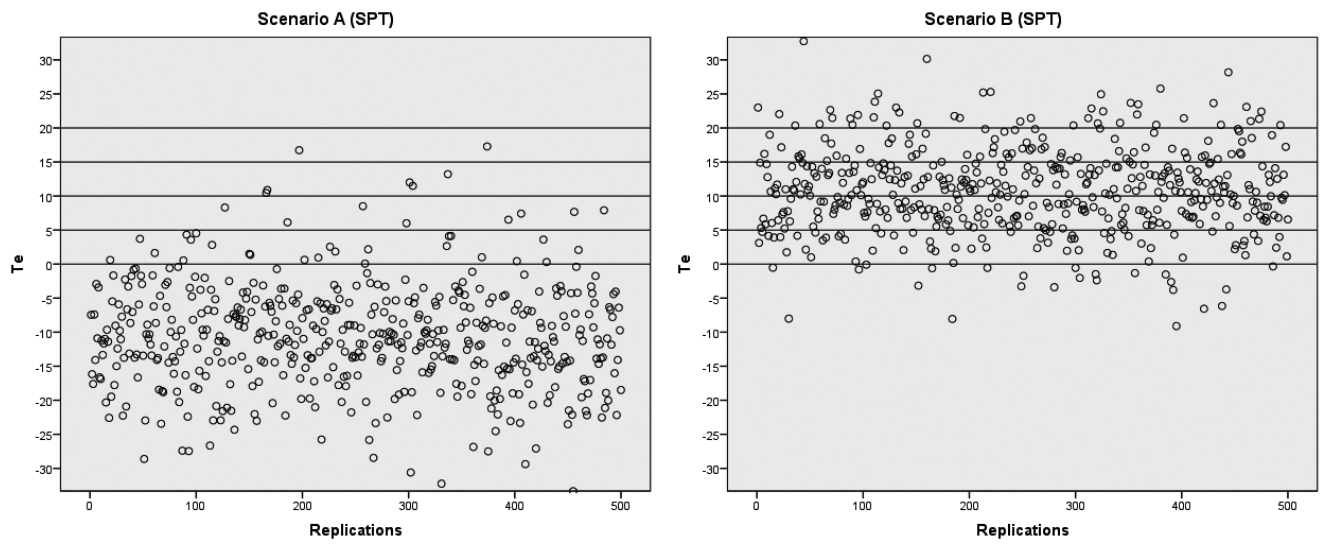

Figure 5 - Comparison of the duration of the replications (expressed as Time in Excess of 120 Minutes, $T e$ ) under the SPT discipline for scenarios A and B.

\subsection{Comparative analysis of restrictions on the arrival process: 80 -minute and 70-minute arrival windows}

Even though the indicator Time in Excess of 120 Minutes $(T e)$ under the SPT queueing discipline is negative on average for scenario $\mathrm{A}$, around $7.8 \%$ of all the replications are completed after the end of the window; in scenario B the percentage is even greater, 95.2\% (see Table 4 and Figure 5). In light of this, it was decided to consider a slack time of some minutes between the time windows so that the last trucks to arrive could be served within the 120 -minute window. For example, if this slack is 40 minutes, then 80-minute arrival windows should be defined for the clients (trucks) to arrive in and 120-minute windows for dispatching, as defined in the operational assumptions. In other words, in the first window, from 7:00 to 9:00 AM, clients should arrive between 7:00 and 8:20 AM; in the next window, from 9:00 to 11:00 AM they should arrive between 9:00 and 10:20 AM; and so on.

In the second simulation, the same scenarios A and B as in the previous simulation were used (corresponding to the different hypotheses for the probability distribution of the arrival times) but with 80-minute and 70-minute arrival windows. The range for the arrival times for the uniform distribution (scenario B) could then be determined.

The choice of expected inter-arrival times in Table 5 for scenario A, corresponding to an exponential distribution, was based on the same criterion explained in Table 1 in Section 3.1. Table 6 shows the simulation parameters (only for the SPT queueing discipline) with the 80-minute and 70-minute arrival windows for scenarios $\mathrm{A}$ and $\mathrm{B}$.

Table 7 shows the values of the indicators in the simulation comparing the performance with 80-minute and 70-minute arrival windows in scenarios A and B. These restricted windows are also compared with the initial 120-minute arrival windows under the SPT queueing discipline in the simulation in the previous section (see also Table 3). 
Table 5 - Relationship between mean values of inter-arrival time (assuming an exponential distribution) and the probability of the thirty trucks not arriving before the end of the 80-minute and 70-minute arrival windows $(P(X<30))(X$ is the number of arrivals in a $Y$-minute window, i.e., before the end of the window).

\begin{tabular}{c|c|c|c|c}
\hline \multirow{2}{*}{} & \multicolumn{4}{|c}{ Inter-arrival time $\sim$ Exponential $(\lambda)$} \\
\cline { 2 - 5 } & Mean inter-arrival time $(1 / \lambda)$ in minutes/arrival \\
\cline { 2 - 5 } & 2.5 & 2.0 & $\mathbf{1 . 7}$ & $\mathbf{1 . 5}$ \\
\hline Y (arrival time window) & \multicolumn{4}{|c}{ Probability that $X$ is less than } \\
& \multicolumn{5}{|c}{$30(X<30))$ in percentage } \\
\hline 80 minutes & $33.81 \%$ & $4.32 \%$ & $\mathbf{0 . 3 2 \%}$ & $0.02 \%$ \\
70 minutes & $62.26 \%$ & $17.70 \%$ & $2.94 \%$ & $\mathbf{0 . 3 8 \%}$ \\
\hline
\end{tabular}

Table 6 - Values of parameters in the second simulation: comparison of the 80-minute and 70-minute arrival windows under the SPT discipline for scenarios A and B.

\begin{tabular}{|c|c|c|c|c|c|}
\hline & & \multicolumn{4}{|c|}{ Scenarios evaluated } \\
\hline Simulation variables & Units & $\mathrm{A}$ & $\mathrm{A}$ & B & B \\
\hline Arrival models & & \multicolumn{2}{|c|}{ Inter-arrival time } & \multicolumn{2}{|c|}{ Arrival time } \\
\hline \multirow{2}{*}{ Distribution } & & \multicolumn{2}{|c|}{ Exponential $(\lambda)$} & \multicolumn{2}{|c|}{ Uniform } \\
\hline & & $\lambda=0.588$ & $\lambda=0.667$ & $U[0,80]$ & $U[0,70]$ \\
\hline \multirow{2}{*}{ Mean } & minutes/arrival or & 1.7 & 1.5 & - & - \\
\hline & minutes & - & - & 40 & 35 \\
\hline \multicolumn{6}{|l|}{ Container move time } \\
\hline \multirow{3}{*}{$\begin{array}{l}\text { Distribution } \\
\text { Mean }(\mu) \\
\text { Standard deviation }(\sigma)\end{array}$} & \multirow{3}{*}{ minutes/container } & \multicolumn{4}{|c|}{ Normal $(N(\mu, \sigma))$} \\
\hline & & 2.5 & 2.5 & 2.5 & 2.5 \\
\hline & & 0.1667 & 0.1667 & 0.1667 & 0.1667 \\
\hline Dispatch time window & minutes & 120 & 120 & 120 & 120 \\
\hline Arrival time window & minutes & 80 & 70 & 80 & 70 \\
\hline
\end{tabular}

The decision to adopt a slack time between the two-hour windows and increase it (giving 120minute, 80-minute and 70-minute arrival windows) and the corresponding reduction in mean inter-arrival time (for example, in the case of scenario A: 2.5, 1.7 and 1.5 minutes/arrival) are determining factors in reducing the likelihood of the dispatch operation exceeding the two-hour window. With these changes the situation is now as follows: the mean values of the variable time in excess of 120 minutes ( $\mathrm{Te}$ ) has decreased, i.e., the difference between the end of the two-hour dispatch window and the end of the dispatch process has increased; the mean number of clients for which servicing finished after the end of the time window has decreased to zero; and, most importantly, the estimated probability of serving the 30 trucks before the end of the time window ( $T e \leq 0$, see Tables 4, 8 and 9 and Figures 5-7) has increased to 1.0 and 0.998 (70-minute arrival windows in scenarios A and B, respectively). However, these two factors have a less beneficial effect on some indicators. For example, the mean total equipment idle time increases because 


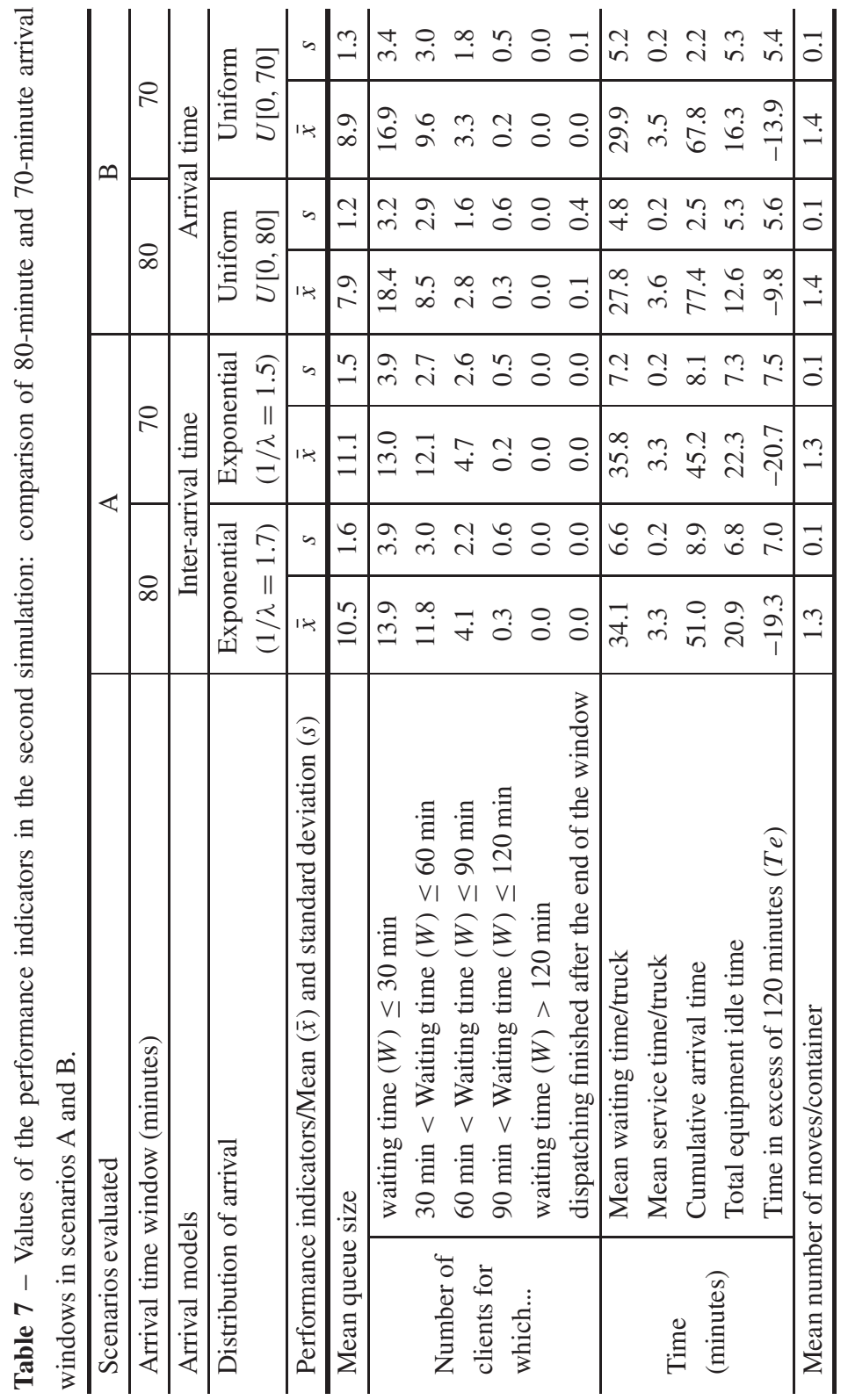


the process finishes earlier, and the average values of the mean waiting time and the mean queue size increase slightly, which is to be expected as the trucks arrive at shorter intervals.

According to Tables 8 and 9 and the graphs in Figures 6 and 7, if a 40-minute or 50-minute slack time between two-hour dispatch windows is considered (i.e., 80-minute and 70-minute arrival windows, respectively), all the clients can be served before the end of the dispatch time window $(T e \leq 0)$ in all the replications in scenario A. In scenario B, however, the percentage of processes that finish before the end of the two-hour dispatch window is $95.6 \%$ and $99.8 \%$ with 80 -minute and 70-minute arrival windows, respectively.

Table 8 - Percentage of replications and the corresponding durations (expressed as $T e$, Time in Excess of 120 Minutes, in five-minute bands) with 80-minute arrival windows under the SPT discipline for scenarios $\mathrm{A}$ and $\mathrm{B}$.

\begin{tabular}{|c|c|c|c|c|c|c|}
\hline Scenarios & \multicolumn{3}{|c|}{ A } & \multicolumn{3}{|c|}{ B } \\
\hline Arrival models & \multicolumn{3}{|c|}{ Exponential $(1 / \lambda=1.7)$} & \multicolumn{3}{|c|}{ Uniform $(U[0,80])$} \\
\hline $\begin{array}{l}\text { Five-minute } \\
\text { bands of } T e\end{array}$ & $\begin{array}{c}\text { Number } \\
\text { of } \\
\text { replications }\end{array}$ & $\begin{array}{l}\text { Percentage } \\
\text { of } \\
\text { replications }\end{array}$ & $\begin{array}{l}\text { Cumulative } \\
\text { percentage of } \\
\text { replications }\end{array}$ & $\begin{array}{c}\text { Number } \\
\text { of } \\
\text { replications }\end{array}$ & $\begin{array}{c}\text { Percentage } \\
\text { of } \\
\text { replications }\end{array}$ & $\begin{array}{c}\text { Cumulative } \\
\text { percentage of } \\
\text { replications }\end{array}$ \\
\hline$T e \leq 0$ & 500 & $100 \%$ & $100 \%$ & 478 & $95.6 \%$ & $95.6 \%$ \\
\hline $0<T e \leq 5$ & 0 & $0.0 \%$ & $100 \%$ & 20 & $4.0 \%$ & $99.6 \%$ \\
\hline $5<T e \leq 10$ & 0 & $0.0 \%$ & $100 \%$ & 1 & $0.2 \%$ & $99.8 \%$ \\
\hline$T e>10$ & 0 & $0.0 \%$ & $100 \%$ & 1 & $0.2 \%$ & $100 \%$ \\
\hline Total (replications) & 500 & $100 \%$ & - & 500 & $100 \%$ & - \\
\hline
\end{tabular}

Table 9 - Percentage of replications and the corresponding durations (expressed as Te, Time in Excess of 120 Minutes, in five-minute bands) with 70-minute arrival windows under the SPT discipline for scenarios $\mathrm{A}$ and $\mathrm{B}$.

\begin{tabular}{|c|c|c|c|c|c|c|}
\hline Scenarios & \multicolumn{3}{|c|}{$\bar{A}$} & \multicolumn{3}{|c|}{ B } \\
\hline Arrival models & \multicolumn{3}{|c|}{ Exponential $(1 / \lambda=1.5)$} & \multicolumn{3}{|c|}{ Uniform $(U[0,70])$} \\
\hline $\begin{array}{l}\text { Five-minute } \\
\text { bands of } T e\end{array}$ & $\begin{array}{c}\text { Number } \\
\text { of } \\
\text { replications }\end{array}$ & $\begin{array}{c}\text { Percentage } \\
\quad \text { of } \\
\text { replications }\end{array}$ & $\begin{array}{l}\text { Cumulative } \\
\text { percentage of } \\
\text { replications }\end{array}$ & $\begin{array}{c}\text { Number } \\
\text { of } \\
\text { replications }\end{array}$ & $\begin{array}{l}\text { Percentage } \\
\text { of } \\
\text { replications }\end{array}$ & $\begin{array}{l}\text { Cumulative } \\
\text { percentage of } \\
\text { replications }\end{array}$ \\
\hline$T e \leq 0$ & 500 & $100 \%$ & $100 \%$ & 499 & $99.8 \%$ & $99.8 \%$ \\
\hline $0<T e \leq 5$ & 0 & $0.0 \%$ & $100 \%$ & 0 & $0.0 \%$ & $99.8 \%$ \\
\hline $5<T e \leq 10$ & 0 & $0.0 \%$ & $100 \%$ & 1 & $0.2 \%$ & $100 \%$ \\
\hline$T e>10$ & 0 & $0.0 \%$ & $100 \%$ & 0 & $0.0 \%$ & $100 \%$ \\
\hline Total (replications) & 500 & $100 \%$ & - & 500 & $100 \%$ & - \\
\hline
\end{tabular}

The performance using 90-minute arrival windows was also investigated, and the corresponding figures (i.e., the replications for which $T e \leq 0$ ) were $99.4 \%$ and $79.6 \%$ for scenarios $\mathrm{A}$ and $\mathrm{B}$, respectively. However, in the interests of space we only describe the results in detail for 80-minute and 70-minute arrival windows. 

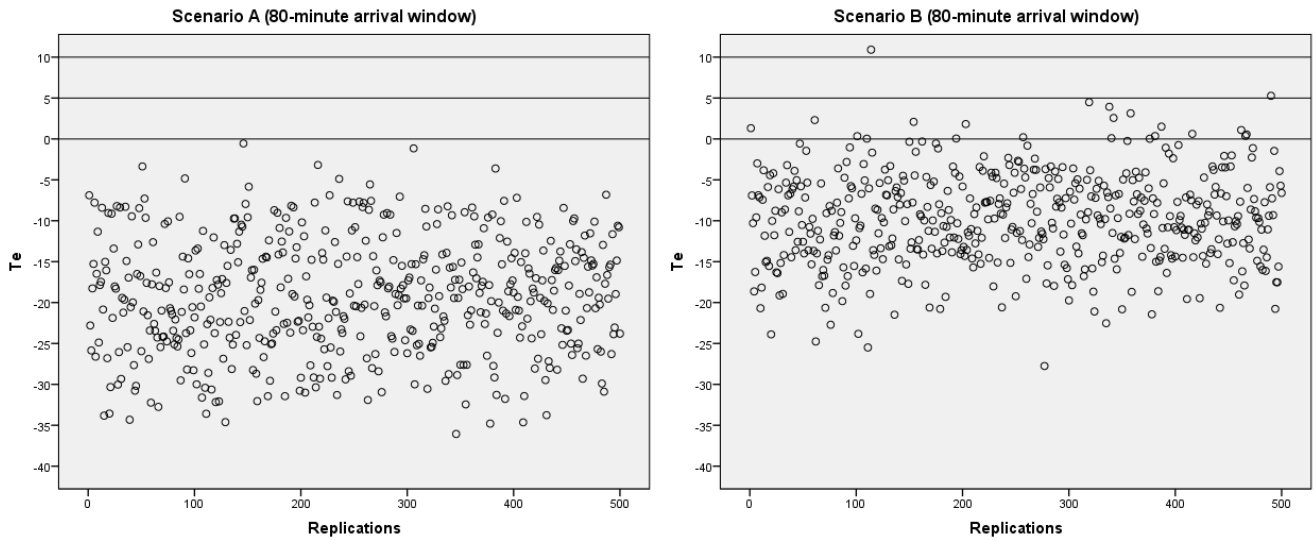

Figure 6 - Comparison of the duration of the replications (Time in Excess of 120 Minutes, $T e$ ) with 80-minute arrival windows under the SPT discipline for scenarios A and B.
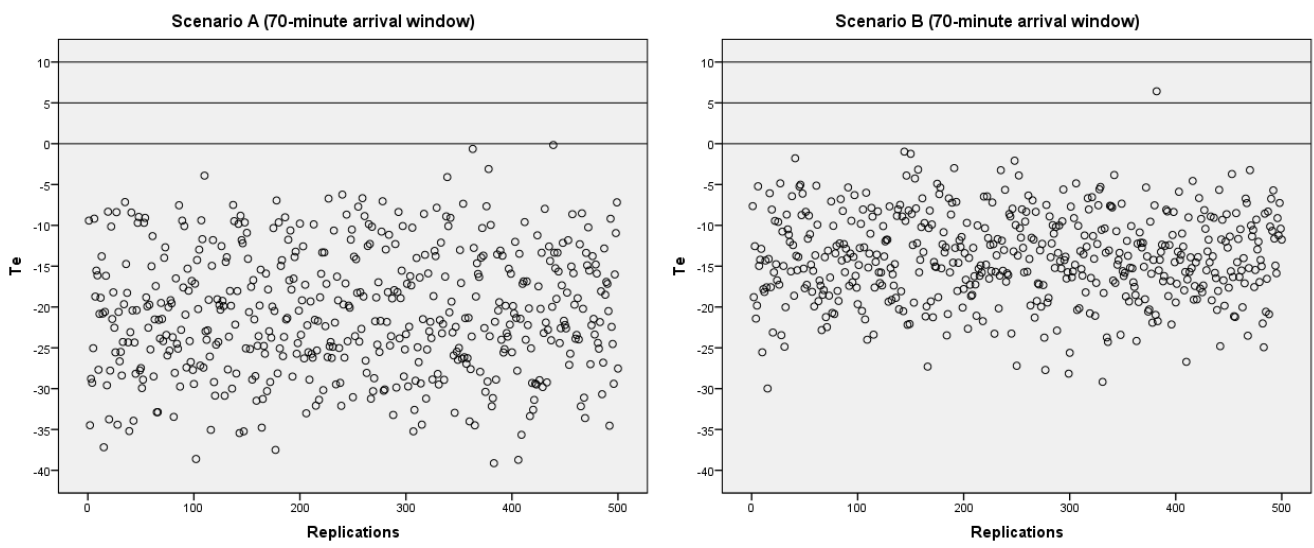

Figure 7 - Comparison of the duration of the replications (Time in Excess of 120 Minutes, $T e$ ) with 70-minute arrival windows under the SPT discipline for scenarios A and B.

These results show that with the adoption of 70-minute arrival windows, in the worst scenario (uniform inter-arrival times), only $1.2 \%$ of the days would have one window in which the servicing would exceed the 2 hours (this can be seen after a quick calculation), but the impact of this would be absorbed (and neutralized, compensated) in the next time window since $99.8 \%$ of the windows finish the service of the 30 trucks before 2 hours (on average, more than 10 minutes before the 2 hours). In the exponentially-distributed inter-arrival time scenario, even 80-minutes arrival windows guarantee the servicing of all 30 trucks within the 2-hour time window. 


\section{CONCLUSION}

Reach stackers, which are used mainly in small terminals, have certain operational advantages, such as flexibility, ability to handle containers, quick transportation of containers over short distances, and also low cost. However, since the use of this kind of equipment has some drawbacks (including, large number of unproductive moves to reach a given container) studies like the present one intend to palliate the disadvantages of these stackers when compared with more sophisticated and expensive equipments by means of certain operational procedures proposed here to provide a more efficient process that can increase the productivity of reach stackers. Below we summarize the main conclusions of this work.

The possible adverse effect (increased waiting time) of the change in the order in which the queues are serviced, regardless of the order of arrival, under the SPT queueing discipline is more than compensated for by the drastic reduction in unproductive moves. As a result, what is in fact observed is a reduction in mean waiting time and mean service time/truck, as well as considerably more promising values for the other indicators. With the SPT queueing discipline there is a much smaller likelihood of dispatching operations exceeding the two-hour time window. It is therefore reasonable to conclude, based on the results of the first simulation, that the SPT queueing discipline yields the best performance for dispatch operations. However, the two scenarios in this simulation (each with different hypotheses) in which the SPT queueing discipline was used failed to ensure that all the clients in a window were served, as only $92.2 \%$ and $4.8 \%$ of the replications finished before the end of the scheduled windows. In view of the uncertainty about arrivals, it was decided to adopt two hypotheses representing extreme scenarios in terms of the distribution of arrivals over time. According to one hypothesis the inter-arrival times have an exponential distribution, resulting in more favorable performance indicators, while according to the other the arrival times have a uniform distribution within the arrival time window.

In the second simulation, which used the SPT queueing discipline, the arrivals of the thirty clients were restricted to the first 80 and 70 minutes in the time window and the hypotheses (scenarios) used were the same as those in the first simulation. The results confirmed the expectation that the smaller the arrival time window and the shorter the mean inter-arrival time, the greater the percentage of replications in which all thirty clients can be served before the end of the scheduled two-hour windows, i.e., the lower the risk of dispatch operations going beyond the end of the time window. Therefore, for both hypotheses, operations with 70-minute arrival windows were most likely to ensure that all the clients were served, as $100 \%$ and $99.8 \%$ of the replications finished before the end of the two-hour window. Nonetheless, as the corresponding figures for operations with an 80 -minute arrival window were $100 \%$ and $95.6 \%$, this restriction is also a viable option. Furthermore, the total idle time and mean waiting time/truck were on average slightly lower than those for the 70-minute arrival window, although the average of the mean service time/truck was approximately the same.

The findings of this study can find application in the many logistic installations where reach stackers are used. Container terminals that make use of processes similar to those described here 
(where, for example, the cost of moving containers may be high) should consider whether the benefits of implementing a system based on SPT discipline outweigh the associated control costs resulting from the need to make a decision according to the particular trucks that are in the queue each time a container is dispatched.

Further studies could usefully investigate hybrid disciplines based on, for example, the smallest number of moves when accessing containers (the SPT queueing discipline) together with upper limits on waiting time. This could result in increased user satisfaction as trucks would not need to spend what clients may consider an excessive amount of time in a queue.

\section{ACKNOWLEDGEMENTS}

We thank two anonymous reviewers and editor for their valuable and constructive comments that improved the paper. This study was financed in part by the Coordenação de Aperfeiçoamento de Pessoal de Nível Superior - Brasil (CAPES) - Finance Code 001 for the first and the fifth authors' research. The second author's work was supported by the CNPq-Brazil, through project 308677/2015-3. Finally, a part of the third author's work was supported by the CNPq-Brazil and FAPERJ through projects 304843/2016-4 and E-26/202.673/2018, respectively.

\section{REFERENCES}

[1] AbrateC (Associação Brasileira dos Terminais de Contêineres de Uso Público). 2018. Estatísticas: movimentação de contêineres (in Portuguese). Available at: http://www. abratec-terminais.org.br/estatisticas. Accessed on April 2nd 2018.

[2] Bearzotti L, Gonzalez R \& Miranda P. 2013. The event management problem in a container terminal. Journal of applied research and technology, 11(1): 95-102.

[3] Bernhofen DM, El-SAhli Z \& KNeller R. 2016. Estimating the effects of the container revolution on world trade. Journal of International Economics, 98: 36-50.

[4] Borgman B, VAn Asperen E \& DeKKer R. 2010. Online rules for container stacking. OR spectrum, 32(3): 687-716.

[5] CARlo HJ, Vis IFA \& Roodbergen KJ. 2014a. Transport operations in container terminals: Literature overview, trends, research directions and classification scheme. European Journal of Operational Research, 236(1): 1-13.

[6] Carlo HJ, Vis IFA \& Roodbergen KJ. 2014b. Storage yard operations in container terminals: Literature overview, trends, and research directions. European Journal of Operational Research, 235(2): 412-430.

[7] Cordeau JF, Gaudioso M, Laporte G \& Moccia L. 2007. The service allocation problem at the Gioia Tauro maritime terminal. European Journal of Operational Research, 176(2): 1167-1184.

[8] CRAinic TG \& Kim KH. 2007. Intermodal Transportation. In: Transportation, Handbooks in Operations Research and Management Science [edited by BARNHART C \& LAPORTE G.], North-Holland, Amsterdam, 467-537.

[9] De Castilho B \& Daganzo CF. 1993. Handling strategies for import containers at marine terminals. Transportation Research-B, 27B(2): 151-166. 
[10] Dekker R, Voogd P \& VAn Asperen E. 2006. Advanced methods for container stacking. OR Spectrum, 28(4): 563-586.

[11] GÜNTHER HO \& KIM KH. 2006. Container terminals and terminal operations. OR Spectrum, 28(4): 437-445.

[12] Hartmann S. 2004. Generating scenarios for simulation and optimization of container terminal logistics. OR Spectrum, 26(2): 171-192.

[13] Junqueira L, Morabito R \& Yamashita DS. 2010. Modelos de otimização para problemas de carregamento de contêineres com considerações de estabilidade e de empilhamento (in Portuguese). Pesquisa Operacional, 30(1): 73-98.

[14] KIм KH \& Kiм HB. 1999. Segregating space allocation models for container inventories in port container terminals. International Journal of Production Economics, 59(1): 415-423.

[15] KozAn E \& CASEY B. 2007. Alternative algorithms for the optimization of a simulation model of a multimodal container terminal. The Journal of the Operational Research Society, 58(9): 1203-1213.

[16] Lee SW, Song DW \& Ducruet C. 2008. A tale of Asia's world ports: the spatial evolution in global hub port cities. Geoforum, 39(1): 372-385.

[17] Levinson M. 2016. The Box: How the Shipping Container Made the World Smaller and the World Economy Bigger, with a new chapter by the author. Princeton University Press.

[18] Moorthy R \& TeO CP. 2006. Berth management in container terminal: the template design problem. OR Spectrum, 28(4): 495-518.

[19] MuRTy KG, LiU J, WAN YW \& Linn R. 2005. A decision support system for operations in a container terminal. Decision Support Systems, 39(3): 309-332.

[20] Novaes AG, Scholz-Reiter B, Silva VMD \& Rosa H. 2012. Long-term planning of a container terminal under demand uncertainty and economies of scale. Pesquisa Operacional, 32(1): 5586.

[21] Pereira ED, Coelho AS, Longaray AA, Machado CMDS \& Munhoz PR. 2018. Metaheuristic analysis applied to the berth allocation problem: case study in a port container terminal. Pesquisa Operacional, 38(2): 247-272.

[22] Rodrigues Junior AT DA S. 2009. A Simulation Model for Picking up Inbound Containers with Time Windows in a Port Terminal. 92 p. M.Sc. Dissertation - Graduate Program in Production Engineering. Department of Industrial Engineering, Pontifical Catholic University of Rio de Janeiro (PUC-Rio), Rio de Janeiro (in Portuguese).

[23] Roy D \& De Koster R. 2012. Optimal Design of Container Terminal Layout. In: Proceedings of International Material Handling Research Colloquium.

[24] SGOURIDIS SP \& ANGELIDES DC. 2002. Simulation-based analysis of handling inbound containers in a terminal. In: Proceedings of the 2002 Winter Simulation Conference, 1716-1724.

[25] Stahlbock R \& Voß S. 2008. Operations research at container terminals: a literature update. $O R$ Spectrum, 30(1): 1-52.

[26] Steenken D, Voß S \& Stahlbock R. 2004. Container terminal operation and operations research - a classification and literature review. OR Spectrum, 26(1): 3-49. 
[27] The World BAnK. 2018. Data: Container port traffic (TEU: 20 foot equivalent units). Available at: http: //data.worldbank. org/indicator/IS.SHP. GOOD.TU/countries. Accessed on April 2nd 2018.

[28] Vacca I, Bierlaire M \& SAlani M. 2007. Optimization at Container Terminals: Status, Trends and Perspectives. In: $7^{\circ}$ Swiss Transport Research Conference.

[29] Vis IfA, De Koster RBM \& Savelsbergh MWP. 2005. Minimum vehicle fleet size under time-window constraints at a container terminal. Transportation Science, 39(2): 249-260.

[30] Vis IFA \& De Koster R. 2003. Transshipment of containers at a container terminal: an overview. European Journal of Operational Research, 147(1): 1-16.

[31] Wilmsmeier G, Tovar B \& SAnchez RJ. 2013. The evolution of container terminal productivity and efficiency under changing economic environments. Research in Transportation Business \& Management, 8: 50-66. 